\title{
Transarterial chemoembolization versus hepatic resection in hepatocellular carcinoma treatment: a meta-analysis
}

\author{
Xin Tian' \\ Ying Dai' \\ Da-qing Wang ${ }^{2}$ \\ Li Zhang' \\ Cheng-guang Sui' \\ Fan-dong Meng' \\ Shen-yi Jiang' \\ Yun-peng Liu' \\ You-hong Jiang'
}

'Molecular Oncology Laboratory of Cancer Research Institute, The First Affiliated Hospital of China Medical University, Shenyang, ${ }^{2} \mathrm{Health}$ and Family Planning Commission of Liaoning Province, People's Republic of China
Correspondence: You-hong Jiang Molecular Oncology Laboratory of Cancer Research Institute, the First Affiliated Hospital of China Medical University, I 55 Nanjing North Street, Heping district, Shenyang I 1000 I, People's Republic of China

Tel +862483282354

Fax +86 2483282473

Email youhongi19@hotmail.com
This article was published in the following Dove Press journal:

Drug Design, Development and Therapy

10 August 2015

Number of times this article has been viewed

Background: A number of cohort studies have compared the outcomes of transarterial chemoembolization (TACE) and hepatic resection (HR) in the treatment of hepatocellular carcinoma (HCC). However, the effect of TACE versus HR remains controversial. Therefore, we conducted a meta-analysis to assess the effectiveness of TACE and HR in HCC treatment.

Materials and methods: PubMed, Embase, Web of Science, Scopus, ClinicalTrials.gov, and Cochrane library were searched from their inception until February 27, 2015 for relevant studies. The literature search was updated on May 25, 2015. Eligible studies were cohort studies comparing the survival outcomes between HCC patients undergoing TACE and HR. The primary outcome was overall survival (OS). Secondary outcomes were the recurrence rate and prognostic factors for OS. The risk ratio (RR) was used for the meta-analysis and was expressed with $95 \%$ confidence intervals (CIs).

Results: This meta-analysis included eleven cohort studies with 6,297 patients, all treated with TACE or HR. Pooled estimates showed that, compared with TACE, HR significantly improved the 3-year OS (RR $=0.77 ; 95 \% \mathrm{CI}, 0.63-0.93 ; P=0.009)$. TACE and HR had similar effects on OS after 1 year $(\mathrm{RR}=0.94 ; 95 \% \mathrm{CI}, 0.86-1.01 ; P=0.103), 2$ years $(\mathrm{RR}=0.50 ; 95 \% \mathrm{CI}, 0.21-1.19$; $P=0.114), 4$ years $(\mathrm{RR}=0.61 ; 95 \% \mathrm{CI}, 0.58-1.10 ; P=0.174)$, and 5 years $(\mathrm{RR}=0.77 ; 95 \% \mathrm{CI}$, $0.59-1.01 ; P=0.06)$. There was no significant difference between the 3 -year $(\mathrm{RR}=1.31 ; 95 \%$ $\mathrm{CI}, 0.65-2.64 ; P=0.457)$ and 5 -year recurrence rates $(\mathrm{RR}=1.14 ; 95 \% \mathrm{CI}, 0.69-1.89 ; P=0.597)$ in the TACE and HR groups. Age $(>65$ vs $\leq 65$ years; hazard ratio $=0.99 ; 95 \% \mathrm{CI}, 0.98-1.00$; $P=0.000$ ), sex (male vs female; hazard ratio $=0.79 ; 95 \% \mathrm{CI}, 0.65-0.96 ; P=0.02$ ), treatment method (TACE vs HR; hazard ratio $=1.90 ; 95 \%$ CI, 1.46-2.46; $P=0.000$ ), and Eastern Cooperative Oncology Group performance score ( $\geq 1$ vs 0 ; hazard ratio =1.69; 95\% CI, 1.22-2.33; $P=0.002$ ) were independent predictors for OS.

Conclusion: This meta-analysis suggests that the TACE and HR likely have similar effects in the treatment of HCC patients in terms of OS and recurrence rate. However, this conclusion should be interpreted cautiously due to the presence of further subgroup analyses with respect to outcomes in patients with different liver statuses (Barcelona Clinic Liver Cancer stage A or stage B).

Keywords: transarterial chemoembolization, hepatic resection, hepatocellular carcinoma, meta-analysis

\section{Introduction}

Hepatocellular carcinoma (HCC) is the third leading cause of cancer-related deaths worldwide, accounting for almost half a million deaths annually. ${ }^{1,2}$ The known causes of $\mathrm{HCC}$ are hepatitis B virus, hepatitis C virus, and chronic alcohol use.,4 With improved periodic surveillance of high-risk patients with chronic hepatitis and 
advances in imaging, more patients are diagnosed with $\mathrm{HCC}$ at an early stage. ${ }^{5}$

For patients with early-stage HCC, curative treatments, including liver transplantation, hepatic resection (HR), and radio frequency ablation (RFA), are currently recommended. ${ }^{6-8}$ HR has been widely used as the standard modality of curative treatment for patients with resectable HCC. ${ }^{8}$ However, it is considered extremely challenging when there is coexisting liver cirrhosis. ${ }^{9,10}$ Liver transplantation is the optimal therapy for HCC, but it is generally limited by the shortage of donors. ${ }^{11,12}$ Consequently, transarterial chemoembolization (TACE) is also widely applied in the treatment of small singlenodule HCC when other curative therapies are not feasible.

TACE is currently recommended for intermediate- or advanced-stage HCC, but it is not recommended for earlystage or resectable HCC. ${ }^{13}$ Some previously published studies have shown that TACE improved long-term survival outcomes in patients with early-stage HCC. ${ }^{14,15}$ In contrast, the results were not observed in another study. ${ }^{16}$ Therefore, we conducted a meta-analysis of all available observational studies to compare survival outcomes for HCC patients undergoing TACE or HR.

\section{Materials and methods Literature search}

We conducted this meta-analysis following the Preferred Reporting Items for Systematic Reviews and Meta-Analyses (PRISMA) guidelines. ${ }^{17}$ To identify relevant studies, we comprehensively searched PubMed, Embase, Web of Science, Scopus, ClinicalTrials.gov, and Cochrane library from their inception until February 27, 2015. The literature search was updated on May 25, 2015. Eligible studies were those comparing the effectiveness of TACE with HR in patients with HCC. The following search terms were used: ("carcinoma, hepatocellular" [MeSH Terms] OR ("carcinoma" [All Fields] AND "hepatocellular" [All Fields]) OR "hepatocellular carcinoma" [All Fields] OR ("hepatocellular" [A1l Fields] AND "carcinoma" [All Fields])) AND hepatic [All Fields] OR liver [All Fields] AND resection [All Fields] AND transarterial [All Fields] AND chemoembolization [All Fields]. The search was limited to trials in human subjects, and no language restriction was applied. Additionally, we also screened the references of identified articles and previous reviews until no new potential articles could be found.

\section{Study selection criteria}

Published studies were included if 1) HCC patients were treated with TACE or HR; 2) the effectiveness of TACE and
HR on HCC were compared; and 3) the studies presented risk ratio (RR) or hazard ratio estimates with $95 \%$ confidence intervals (95\% CIs). When multiple publications from the same trial were available, we selected only the publication with the most information or latest data. Narrative reviews, systematic reviews, letters, comments, or studies unrelated to our topics were excluded.

\section{Data extraction and quality assessment}

A structured questionnaire was used for data extraction. Two investigators (YD and YHJ) independently performed the eligibility evaluation, data extraction, and quality assessment. Disagreements were resolved through discussion and consensus. Data extracted from each study were as follows: first author's name, year of publication, study population, range for follow-up, hepatitis status, overall survival (OS) rate, recurrence rate, and hazard ratio estimates with 95\% CIs.

The methodological quality of the studies included in the meta-analysis was assessed using the 9-star system of the modified Newcastle-Ottawa Scale. ${ }^{18}$ The scale consists of three items describing patient selection, comparability of the TACE and HR groups, and assessment of outcomes of interest. The full score was 9 , and articles with quality score greater than or equal to 6 were considered to be of high quality.

\section{Statistical analysis}

We compared the overall effectiveness of TACE and HR in the treatment of HCC based on the data from the included studies. For the dichotomous variables (ie, the OS rate, recurrence rate), the number of events and the total number of patients were extracted from the included studies. For timeto-event variables (ie, cumulative OS), hazard ratios with 95\% CIs were directly extracted from the included studies. Finally, the RR and hazard ratio of each study were pooled using a fixed-effects model (Mantel-Haenszel method) ${ }^{19}$ or a randomized-effects model (DerSimonian-Laird method). ${ }^{20}$ Heterogeneity between studies was assessed using the $I^{2}$ statistic. An $I^{2}$ value of $<25 \%, \sim 50 \%, \sim 75 \%$, or $\sim 100 \%$ was considered to have no, low, moderate, or high heterogeneity, respectively. ${ }^{21}$ When substantial heterogeneity existed, a randomized-effects model was used to summarize the pooled data. Otherwise, a fixed-effect model was used.

The presence of publication bias was evaluated by using the Begg and Egger tests. ${ }^{22,23}$ A $P$-value $<0.05$ was judged as statistically significant. All statistical analyses were performed using STATA version 12.0 (StataCorp LP, College Station, TX, USA). 


\section{Results}

\section{Identification of eligible studies}

The initial search yielded 1,519 relevant citations from PubMed, Embase, Web of Science, Scopus, ClinicalTrials. gov, and Cochrane library. Of these, 789 articles were excluded due to duplicate records and 677 after review of the title and abstract (Figure 1). Therefore, 53 articles were identified for full-text information analysis, and 42 studies were excluded because they did not report usable or sufficient data for analysis. Finally, eleven studies involving 6,297 patients ${ }^{16,24-33}$ who met the inclusion criteria were included in this meta-analysis.

\section{Characteristics of eligible studies}

The baseline characteristics of the patients in the included studies are presented in Table 1. The eleven articles were published between 1992 and 2014. Of the eleven trials, four were done in Taiwan, People's Republic of China; $;^{16,25,26,33}$ two in each of mainland People's Republic of China ${ }^{28,30}$ and Japan, ${ }^{29,32}$ and one each in Korea, ${ }^{24}$ Germany, ${ }^{27}$ and France. ${ }^{31}$ The number of patients ranged from 72 to 1,296 , with a total of 6,297 patients. In a study conducted by Yang et $\mathrm{al}^{24}$ the authors used inverse probability weighting and a regression method to balance underlying liver function in the treatment group. In three other studies, ${ }^{25,28,33}$ propensity score matching was performed to reduce the effects of selection bias. Most individual studies were matched or adjusted for a wide range of potential confounders, including patient age, severity of liver disease, alpha-fetoprotein levels, and tumor size. The quality of the nonrandomized studies was evaluated according to a modification of the Newcastle-Ottawa Scale. The scores ranged from 6 to 9 , indicating that these studies were of high quality (Table 1).

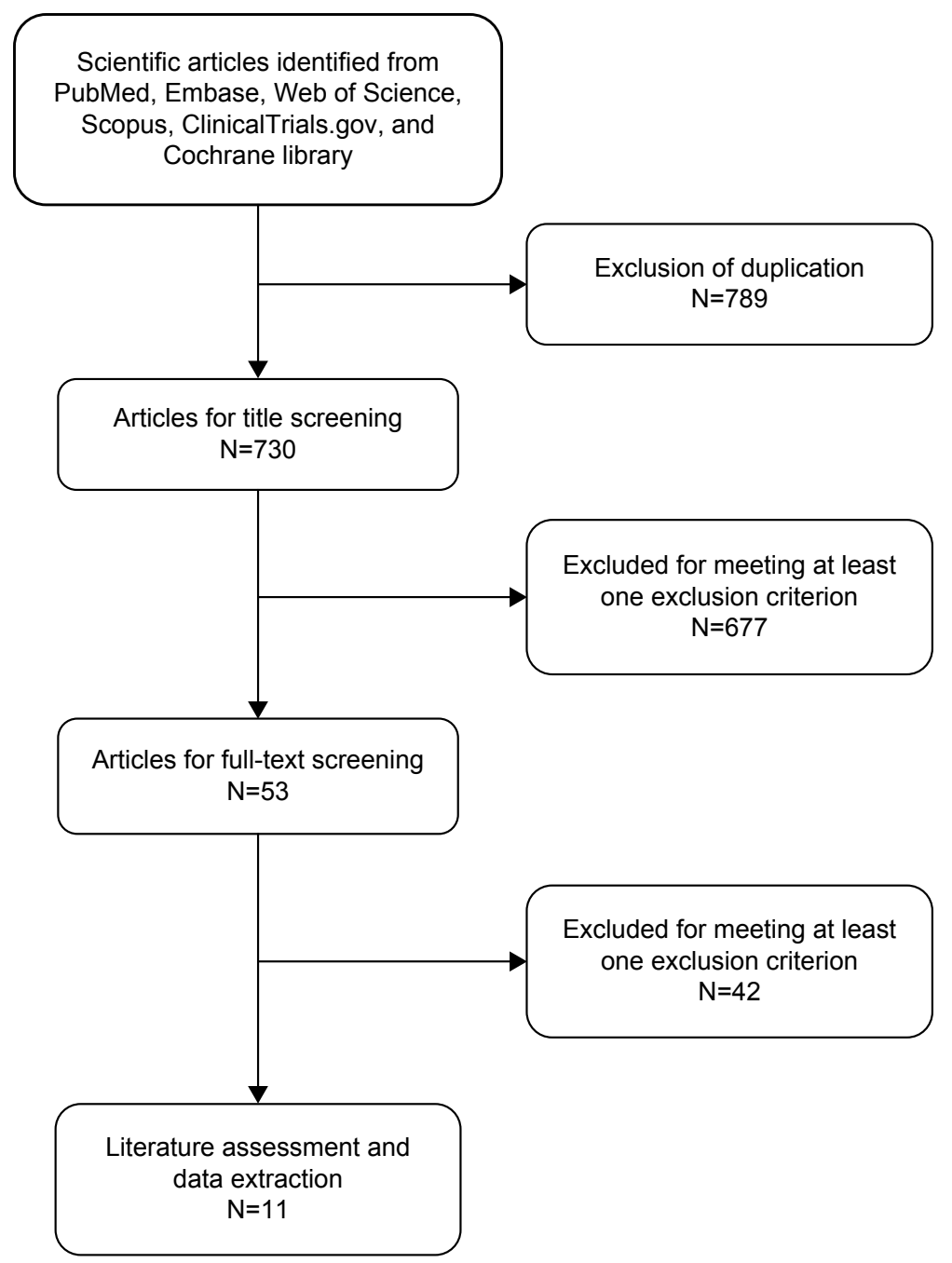

Figure I Eligibility of studies for inclusion in the meta-analysis. 


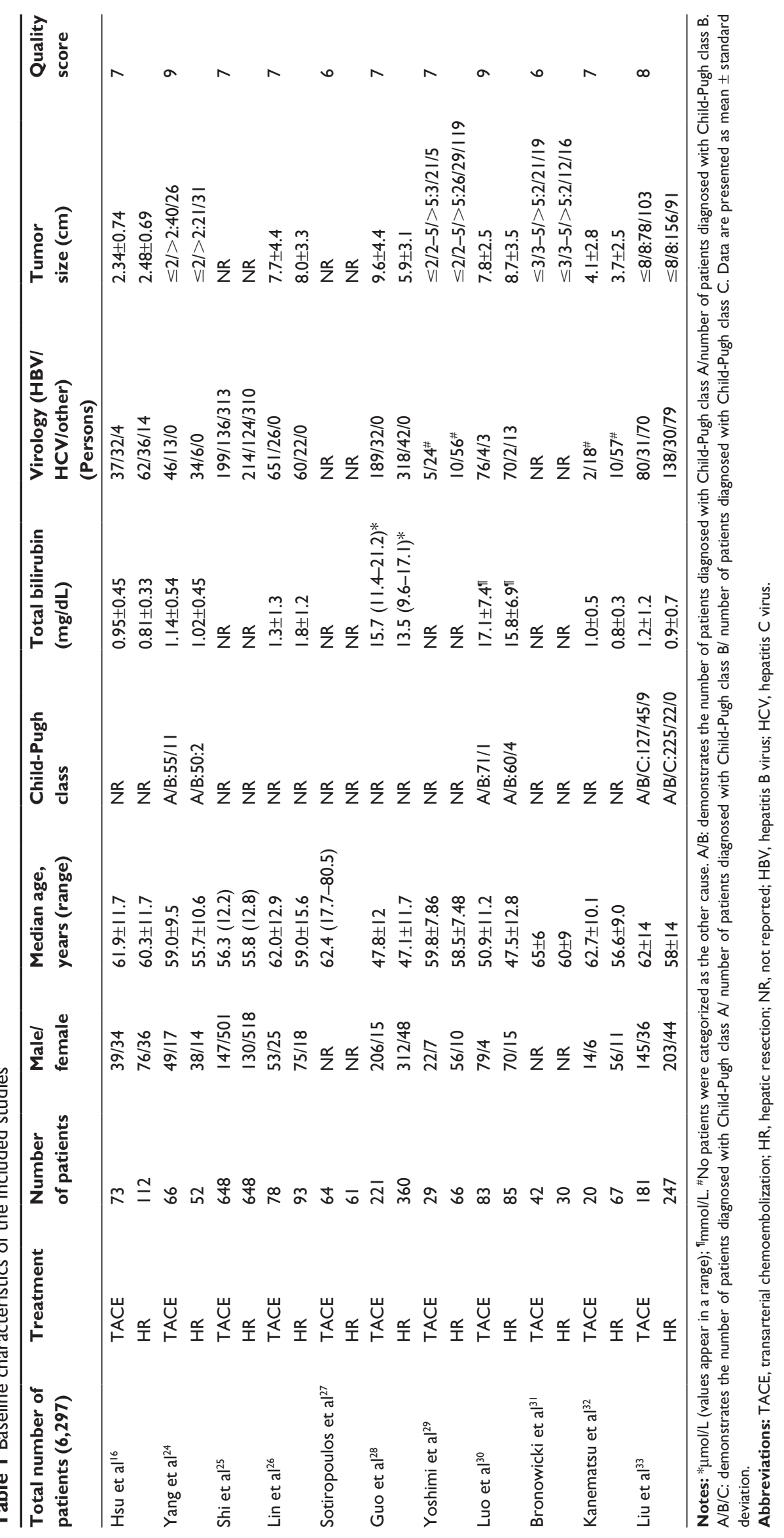




\section{Overall survival}

\section{One-year survival}

Ten studies reported the data of 1-year survival rates. ${ }^{16,24-30,32,33}$ Of them, seven studies ${ }^{16,24,25,27,29,30,32}$ showed that the 1-year survival rate was similar between the two groups. The remaining three studies ${ }^{26,28,33}$ demonstrated that the 1-year survival rate was significantly higher in the HR group than in the TACE group. Using a random-effects model, the aggregated results suggested that the TACE was not significantly associated with higher 1-year survival compared to $\mathrm{HR}(\mathrm{RR}=0.94 ; 95 \% \mathrm{CI}, 0.86-1.01 ; P=0.103$ ) (Figure 2$)$. The Egger test $(P=0.474)$ and Begg test $(P=0.236)$ revealed no publication bias.

\section{Two-year survival}

Among the eleven studies included in this meta-analysis, four reported the data of 2-year survival rates. ${ }^{26,27,29,32} \mathrm{Of}$ them, two studies ${ }^{29,32}$ showed that the 2-year survival rate was similar between the two groups. The remaining two studies $^{26,27}$ demonstrated that the 2-year survival rate was significantly higher in the HR group than in the TACE group. Using a random-effects model, the pooled estimates revealed that patients in the TACE group did not have higher 2-year survival rate than those in the $\mathrm{HR}$ group $(\mathrm{RR}=0.50$; 95\% CI, 0.21-1.19; $P=0.114$ ) (Figure 3). As the number of included studies was less than five, publication bias was not assessed.

\section{Three-year survival}

All the studies reported the data of 3-year survival rates. ${ }^{16,24-33}$ Of them, seven studies ${ }^{16,25,26,29-32}$ showed that the 3-year survival rate was similar between the two groups. The remaining four studies ${ }^{24,27,28,33}$ demonstrated that the 3-year survival rate was significantly higher in the HR group than in the TACE group. The aggregated results suggested that patients treated with HR had a higher 3-year survival rate compared with those treated with TACE $(\mathrm{RR}=0.77 ; 95 \% \mathrm{CI}, 0.63-0.93$; $P=0.009)$ (Figure 4). An Egger test $(P=0.107)$ and a Begg test $(P=0.533)$ revealed no publication bias.

\section{Four-year survival}

Among the eleven studies included in this meta-analysis, only three studies reported the data of 4-year survival rates. ${ }^{27,29,32} \mathrm{Of}$ them, two studies ${ }^{29,32}$ showed that the 4-year survival rate was similar between the two groups. The remaining study ${ }^{27}$ demonstrated that the 4-year survival rate was significantly higher in the HR group than in the TACE group. Using a random-effects model, the pooled estimates revealed that the patients in the TACE group did not have a higher 4-year survival rate than those in the HR group $(\mathrm{RR}=0.61 ; 95 \% \mathrm{CI}, 0.34-1.10 ; P=0.174)$ (Figure 5). As the number of eligible studies for meta-analysis was less than five, publication bias was not assessed.

\section{Five-year survival}

Of the eleven studies included in this meta-analysis, ten studies reported the data of 5-year survival rates. ${ }^{16,24,25,27-33}$

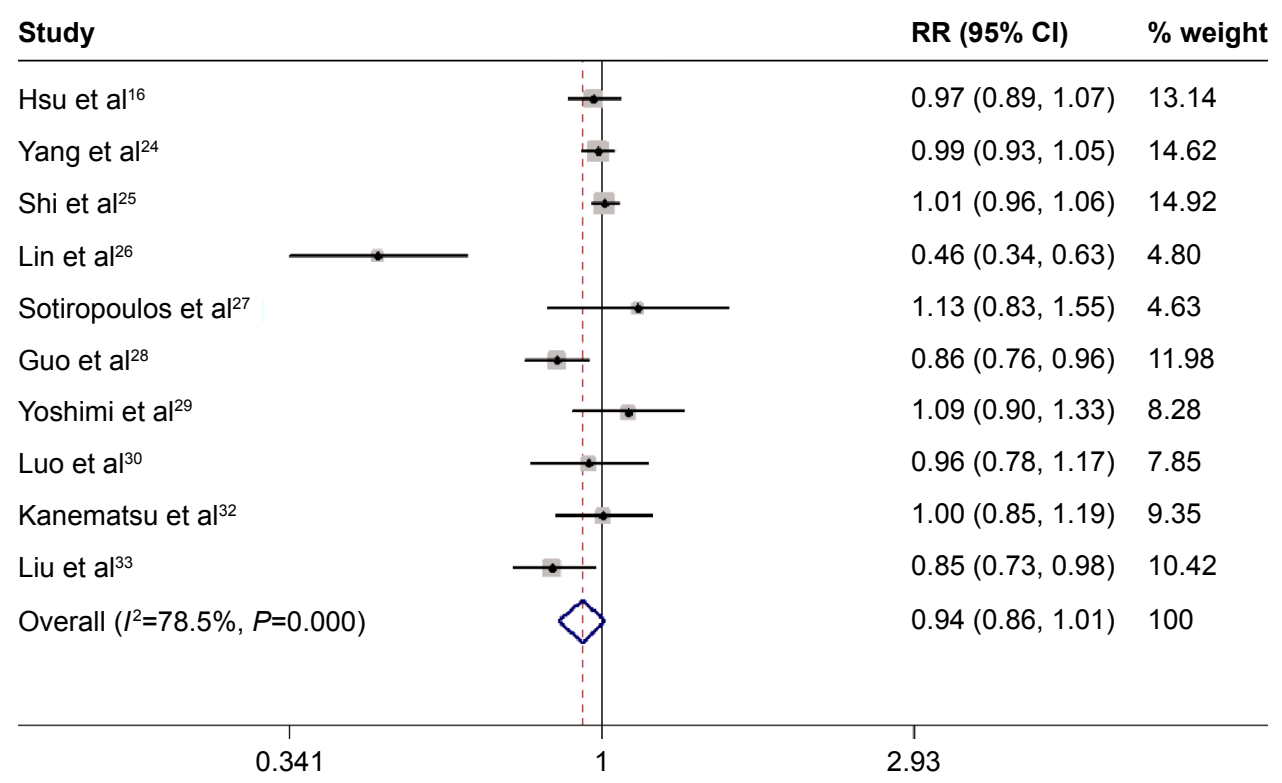

Figure 2 TACE versus HR for HCC in terms of I-year overall survival rate.

Notes: Boxes are the relative risk estimates from each study; the horizontal bars are $95 \%$ Cls. The size of the box is proportional to the weight of the study in the pooled analysis. Weights are from random effects analysis.

Abbreviations: TACE, transarterial chemoembolization; $\mathrm{HR}$, hepatic resection; $\mathrm{HCC}$, hepatocellular carcinoma; $\mathrm{Cl}$, confidence interval; $\mathrm{RR}$, risk ratio. 


\begin{tabular}{|c|c|c|}
\hline Study & RR (95\% Cl) & $\%$ weight \\
\hline Lin et $\mathrm{al}^{26}$ & $0.09(0.03,0.23)$ & 20.84 \\
\hline Sotiropoulos et $\mathrm{al}^{27}$ & $0.53(0.32,0.87)$ & 25.62 \\
\hline Yoshimi et al ${ }^{29}$ & $1.17(0.85,1.60)$ & 26.98 \\
\hline Kanematsu et $\mathrm{al}^{32}$ & $0.76(0.52,1.11)$ & 26.57 \\
\hline Overall $\left(I^{2}=93.0 \%, P=0.000\right)$ & $0.50(0.21,1.19)$ & 100 \\
\hline 0.0334 & 29.9 & \\
\hline
\end{tabular}

Figure 3 TACE versus HR for HCC in terms of 2-year overall survival rate.

Notes: Boxes are the relative risk estimates from each study; the horizontal bars are $95 \%$ Cls. The size of the box is proportional to the weight of the study in the pooled analysis. Weights are from random effects analysis.

Abbreviations: TACE, transarterial chemoembolization; $\mathrm{HR}$, hepatic resection; $\mathrm{HCC}$, hepatocellular carcinoma; $\mathrm{Cl}$, confidence interval; $\mathrm{RR}$, risk ratio.

Of them, six studies ${ }^{16,25,27,29-31}$ showed that 5-year survival rate was similar between the two groups. The remaining four studies $24,28,32,33$ demonstrated that 5-year survival was significantly higher in the HR group than in the TACE group. Using a random-effects model, the pooled studies revealed that patients in the TACE group did not have a higher 5-year survival rate than those in the $\mathrm{HR}$ group $(\mathrm{RR}=0.77 ; 95 \% \mathrm{CI}$, 0.59-1.01; $P=0.06)$ (Figure 6). An Egger test $(P=0.080)$ and a Begg test $(P=1.000)$ revealed no publication bias.

\section{Recurrence}

Among the eleven studies included in this meta-analysis, three studies reported the data of 3-year recurrence rate. ${ }^{16,24,31}$ Two studies ${ }^{16,24}$ showed that patients treated with TACE appeared to have a higher 3-year recurrence rate than those treated with HR. The other study ${ }^{31}$ indicated that the 3-year recurrence rate of TACE was lower than that of HR. The pooled results indicated that the 3-year recurrence rate was not significantly different between the TACE and HR groups $(\mathrm{RR}=1.31 ; 95 \% \mathrm{CI}, 0.65-2.64 ; P=0.457$ ) (Figure 7).

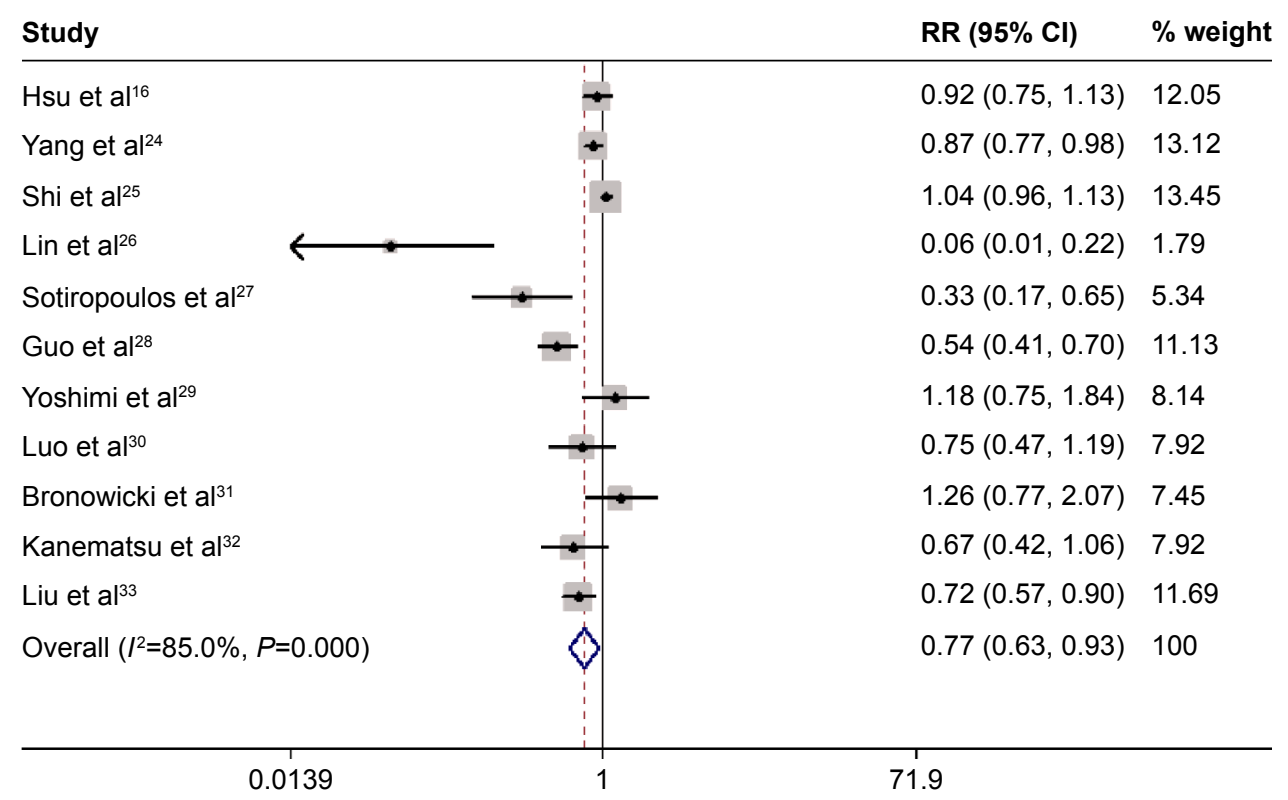

Figure 4 TACE versus HR for HCC in terms of 3-year overall survival rate.

Notes: Boxes are the relative risk estimates from each study; the horizontal bars are $95 \%$ Cls. The size of the box is proportional to the weight of the study in the pooled analysis. Weights are from random effects analysis.

Abbreviations: TACE, transarterial chemoembolization; $\mathrm{HR}$, hepatic resection; $\mathrm{HCC}$, hepatocellular carcinoma; $\mathrm{Cl}$, confidence interval; RR, risk ratio. 


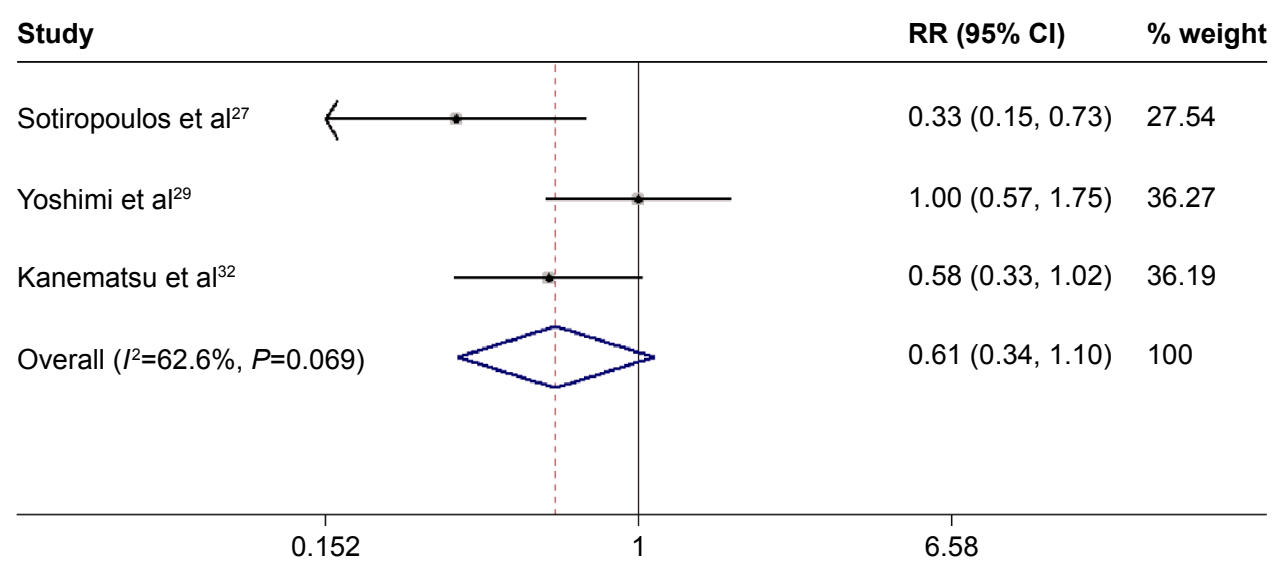

Figure 5 TACE versus HR for HCC in terms of 4-year overall survival rate.

Notes: Boxes are the relative risk estimates from each study; the horizontal bars are $95 \% \mathrm{Cls}$. The size of the box is proportional to the weight of the study in the pooled analysis. Weights are from random effects analysis.

Abbreviations: TACE, transarterial chemoembolization; HR, hepatic resection; HCC, hepatocellular carcinoma; $\mathrm{Cl}$, confidence interval; $\mathrm{RR}$, risk ratio.

Three studies ${ }^{16,24,31}$ presented the data of 5-year recurrence rate. Of them, two studies ${ }^{16,24}$ showed that the 5-year recurrence rate was significantly higher in the TACE group than in the HR group. The remaining study ${ }^{31}$ suggested that the 5-year recurrence rate of TACE was lower than that of HR. The pooled analysis showed that the 5-year recurrence rate was not significantly different between the two groups $(\mathrm{RR}=1.14 ; 95 \%$ CI, 0.69-1.89; $P=0.597)$ (Figure 7).

\section{Prognostic factors for OS}

Five studies ${ }^{16,24-26,33}$ reported the data of prognostic factors for OS. The pooled estimates showed that age $(>65$ vs $\leq 65$ years; hazard ratio $=0.99 ; 95 \%$ CI, $0.98-1.00$;
$P=0.000$ ), sex (male vs female; hazard ratio $=0.79 ; 95 \% \mathrm{CI}$, $0.65-0.96 ; P=0.02$ ), treatment method (TACE vs HR; hazard ratio $=1.90 ; 95 \% \mathrm{CI}, 1.46-2.46 ; P=0.000$ ), and Eastern Cooperative Oncology Group performance score ( $\geq 1$ vs 0 ; hazard ratio $=1.69 ; 95 \% \mathrm{CI}, 1.22-2.33 ; P=0.002)$ were independent predictors of OS (Table 2).

\section{Discussion}

To the best of our knowledge, this meta-analysis is the first to compare survival outcomes and prognostic factors for HCC patients receiving TACE or HR. Meta-analysis of all included studies showed that the 1-year, 2-year, 4-year, and 5-year OS rates and 3-year and 5-year recurrence rates

\begin{tabular}{|c|c|c|c|}
\hline Study & & $\operatorname{RR}(95 \% \mathrm{Cl})$ & $\%$ weight \\
\hline Hsu et al ${ }^{16}$ & - & $0.91(0.69,1.20)$ & 12.41 \\
\hline Yang et $\mathrm{al}^{24}$ & & $0.79(0.67,0.92)$ & 13.55 \\
\hline Shi et $\mathrm{al}^{25}$ & $\mp$ & $1.27(1.14,1.40)$ & 13.93 \\
\hline Sotiropoulos et $\mathrm{al}^{27}$ & & $0.48(0.21,1.10)$ & 6.00 \\
\hline Guo et $\mathrm{al}^{28}$ & & $0.45(0.31,0.65)$ & 11.24 \\
\hline Yoshimi et al ${ }^{29}$ & & $1.20(0.61,2.38)$ & 7.46 \\
\hline Luo et $\mathrm{al}^{30}$ & & $0.82(0.46,1.47)$ & 8.53 \\
\hline Bronowicki et al ${ }^{31}$ & & $1.10(0.65,1.84)$ & 9.32 \\
\hline Kanematsu et al ${ }^{32} \leftarrow$ & & $0.36(0.15,0.89)$ & 5.48 \\
\hline Liu et $\mathrm{al}^{33}$ & & $0.59(0.44,0.80)$ & 12.08 \\
\hline Overall $\left(I^{2}=87.0 \%, P=0.000\right)$ & & $0.77(0.59,1.01)$ & 100 \\
\hline
\end{tabular}

Figure 6 TACE versus HR for HCC in terms of 5-year overall survival rate.

Notes: Boxes are the relative risk estimates from each study; the horizontal bars are $95 \% \mathrm{Cls}$. The size of the box is proportional to the weight of the study in the pooled analysis. Weights are from random effects analysis.

Abbreviations: TACE, transarterial chemoembolization; $\mathrm{HR}$, hepatic resection; $\mathrm{HCC}$, hepatocellular carcinoma; $\mathrm{Cl}$, confidence interval; $\mathrm{RR}$, risk ratio. 


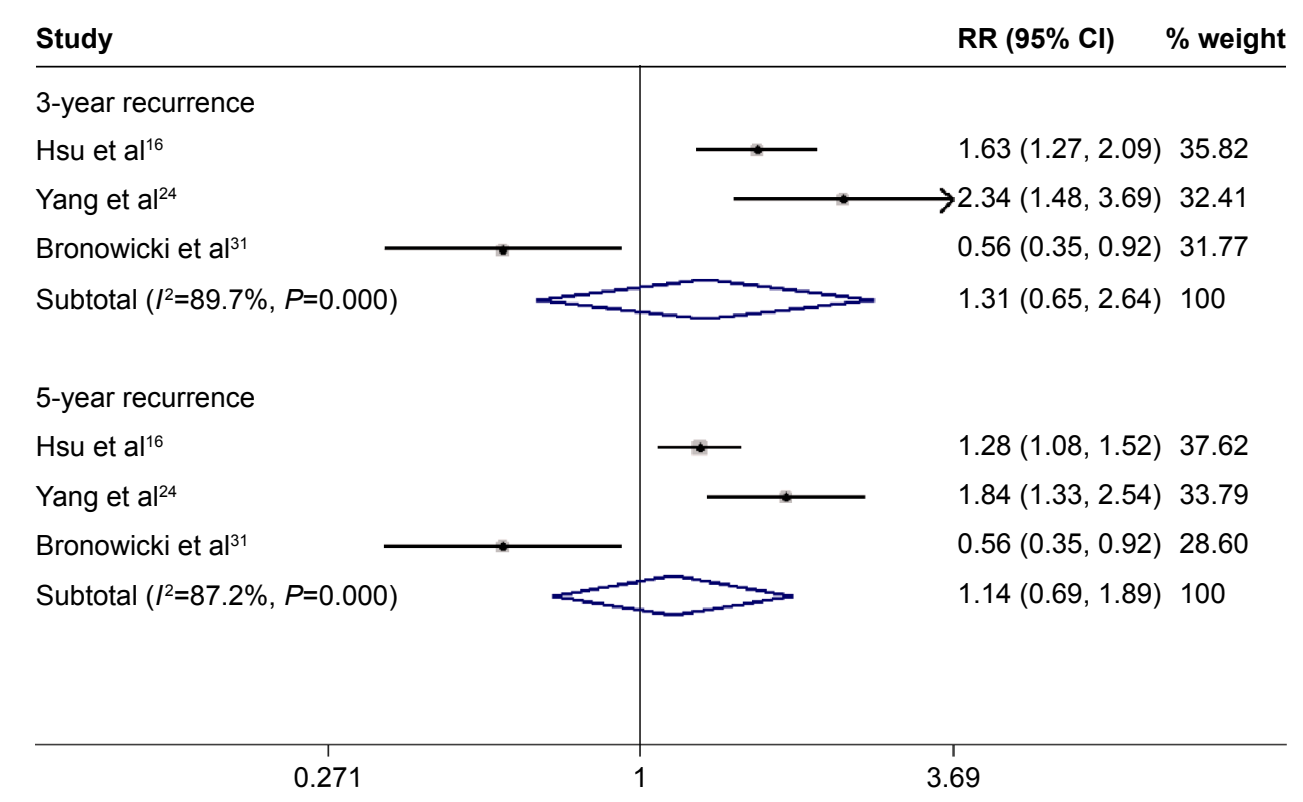

Figure 7 TACE versus HR for HCC in terms of 3-year recurrence rate and 5-year recurrence rate.

Notes: Boxes are the relative risk estimates from each study; the horizontal bars are $95 \% \mathrm{Cls}$. The size of the box is proportional to the weight of the study in the pooled analysis. Weights are from random effects analysis.

Abbreviations: TACE, transarterial chemoembolization; HR, hepatic resection; $\mathrm{HCC}$, hepatocellular carcinoma; $\mathrm{Cl}$, confidence interval; $\mathrm{RR}$, risk ratio.

were not significantly different in HCC patients treated with TACE or HR. However, the 3-year OS rate of HCC patients undergoing HR was higher than that of those undergoing TACE (RR $=0.77 ; 95 \%$ CI, 0.63-0.93; $P=0.009)$. Our findings demonstrated similar benefits with TACE and HR on survival and recurrence.

HR is recognized as the standard modality of curative treatment for patients with resectable HCC. ${ }^{8}$ Also, HR has better recurrence-free survival rates than that of TACE, which

Table 2 Meta-analysis of the prognostic factors for overall survival

\begin{tabular}{|c|c|c|c|}
\hline Prognostic factors & HR & $95 \% \mathrm{Cl}$ & $P$-value \\
\hline Age, years ( $>65$ vs $\leq 65)$ & 0.99 & $0.98-1.00$ & 0.000 \\
\hline $\operatorname{Sex}(M$ vs $F)$ & 0.79 & $0.65-0.96$ & 0.020 \\
\hline HBV (+ vs -) & 1.10 & $0.92-1.30$ & 0.295 \\
\hline $\mathrm{HCV}(+\mathrm{vs}-)$ & 1.00 & $0.86-1.20$ & 0.820 \\
\hline Platelet, $10^{3} / \mu \mathrm{L}(<10$ vs $\geq 10)$ & 1.24 & $0.89-1.72$ & 0.212 \\
\hline Albumin, g/dL $(<4$ vs $\geq 4)$ & 1.16 & $0.60-2.24$ & 0.664 \\
\hline Total bilirubin, $\mathrm{mg} / \mathrm{dL}(<|.2 \mathrm{vs} \geq| .2)$ & 0.95 & $0.46-1.93$ & 0.876 \\
\hline ALT, $U / L(<80$ vs $\geq 80)$ & 0.92 & $0.67-1.30$ & 0.621 \\
\hline AFP, ng/mL (>200 vs $\leq 200)$ & 1.27 & $0.73-2.19$ & 0.394 \\
\hline Tumor size $(>2 \mathrm{~cm}$ vs $\leq 2 \mathrm{~cm})$ & 1.36 & $0.7 I-2.6 I$ & 0.358 \\
\hline Tumor number ( 2 or 3 vs I) & 1.01 & $0.38-2.68$ & 0.981 \\
\hline Treatment method (TACE vs HR) & 1.90 & $1.46-2.46$ & 0.000 \\
\hline Liver cirrhosis & 1.46 & $0.43-4.94$ & 0.540 \\
\hline ECOG PS ( $\geq I$ vs 0$)$ & 1.69 & $1.22-2.33$ & 0.002 \\
\hline
\end{tabular}

Abbreviations: $\mathrm{HR}$, hazard ratio; $\mathrm{Cl}$, confidence interval; $\mathrm{M}$, male; $\mathrm{F}$, female; $\mathrm{HBV}$, hepatitis B virus; HCV, hepatitis C virus; TACE, transarterial chemoembolization; ECOG PS, Eastern Cooperative Oncology Group performance score; ALT, alanine aminotransferase; AFP, alpha-fetoprotein. may affect the life quality of patients. However, the drawback of HR is that it is a more invasive treatment with surgical complications, and it is considered extremely challenging when the liver cirrhosis coexists. ${ }^{9,10}$ TACE is currently recommended for intermediate or advanced-stage HCC, but it is not recommended for early-stage or resectable HCC. ${ }^{13}$ When TACE is used as an initial treatment, special care should be taken to obtain a complete response, and surveillance for tumor recurrence should be undertaken.

TACE is considered as a palliative treatment for HCC. The extensive tumor necrosis induced by TACE treatment could occur in $\sim 30 \%-50 \%$ of patients, while only less than $2 \%$ of patients achieved a complete response. ${ }^{34}$ According to previous studies, the 5-year survival rates of TACE in unresectable HCC patients were about $6 \%-19 \% .{ }^{35-37}$ However, in this study, we found a higher 5-year survival rate, $11.0 \%-74.2 \%$, than previous studies. Yang et al ${ }^{24}$ reported a retrospective cohort study comparing the effectiveness of TACE, RFA, and HR in patients with small single-nodule HCC. In their study, they found a 5 -year OS rate of $74.2 \%$ in the TACE group, $86.6 \%$ in the RFA group, and $93.6 \%$ in the HR group $(P=0.023)$. However, when the inverse probability weighting was used, the weighted OS rates among the three groups were similar. The 5 -year OS was $80.7 \%$ with TACE, $87.6 \%$ with RFA, and $85.6 \%$ with HR $(P=0.834) .{ }^{24}$ Therefore, the authors concluded that TACE is an effective treatment and is comparable to HR and RFA in patients with small single-nodule HCC. 
In contrast, HR is the current standard treatment for early-stage $\mathrm{HCC}$ and allows a better long-term survival rate. ${ }^{13}$ Moreover, recurrence is lower with HR than with TACE, which may affect the patient's quality of life. In a propensity score-matched study, HCC patients with Barcelona Clinic Liver Cancer (BCLC) stage A were treated with TACE or HR. The 3-year and 5-year OS rates were $24.1 \%$ and $13.7 \%$ after TACE, respectively, and $44.8 \%$ and $30.2 \%$ after HR, respectively. ${ }^{28}$ Therefore, it was assumed that HR was associated with higher survival rates than TACE in patients with BCLC stage A and stage B. However, in this study, these survival benefits were not observed when HR was applied in HCC patients with Child class A or class B. Of the included studies, two trials conducted by Hsu et $\mathrm{al}^{16}$ and Yoshimi et al ${ }^{29}$ showed that HR did not provide significantly better survival over TACE in HCC patients. These differences in survival outcomes between TACE and HR might be attributed to the liver status and other characteristics of enrolled patients.

One of the studies included in our meta-analysis reported an interesting outcome for recurrence. Among the three studies reporting the recurrence rate, ${ }^{16,24,31}$ two $^{16,24}$ showed that TACE resulted in a significantly higher recurrence rate than HR. In the study by Hsu et $\mathrm{al}^{16}$ the 3 -year and 5-year recurrence rates were $72.6 \%$ versus $44.6 \%$ for TACE, respectively, and $83.6 \%$ versus $65.2 \%$ for HR, respectively. In the study by Yang et $\mathrm{al}^{24}$ the 3-year and 5-year recurrence rates were $68.9 \%$ versus $29.4 \%$ for TACE, respectively, and $82.8 \%$ versus $45.1 \%$ for HR, respectively. However, another study ${ }^{31}$ showed that patients treated with TACE had lower 3-year and 5-year recurrence rates (3-year and 5-year recurrence rates, 35\%) than those treated with HR (3-year and 5-year recurrence rates, $64 \%$ ). The reason HCC patients treated with TACE in the latter study had a lower recurrence rate than those treated with HR remains unknown.

This meta-analysis has several potential limitations. First, we acknowledge that this meta-analysis was performed based on cohort studies rather than randomized controlled trials (RCTs). RCTs are regarded as the most efficient and highlevel evidence for clinical research, whereas cohort studies provided relatively low-level clinical evidence, which may lead to selection bias. Although an RCT would allow unbiased comparison of treatment effectiveness, it is not easy to execute the TACE and HR procedures using a randomized controlled design due to current practice guidelines and ethical issues. Second, there was significant heterogeneity between studies in the overall analysis. This might be attributed to the baseline patient characteristics (age, cause of liver disease, liver function, tumor size, and tumor number), sample size, and study quality. These factors may have a potential impact on our final results. Third, due to the lack of sufficient data, we were unable to perform subgroup analyses to compare the effect of TACE and HR on patients with different disease statuses.

In summary, the current meta-analysis shows that TACE and HR seem to have similar effects in $\mathrm{HCC}$ treatment with respect to OS and recurrence. Although further multicenter, well-designed RCTs are needed to verify these findings, TACE could be an alternative treatment in patients with early-stage or resectable HCC. More notably, insufficient data limits further subgroup analyses and comparison of the treatment effect between TACE and HR by liver status (BCLC stage A or stage B). Further research should focus on resolving this issue.

\section{Acknowledgments}

This study was supported by grants from the National Natural Science Foundation of China (81202955), Shenyang Municipal Science and Technology Program Funded Projects (F 12-278-6-09), Liaoning Medical Peak Construction Engineering Projects (2012029), and Liaoning Science and Technology Projects (2011415052-3).

\section{Disclosure}

The authors report no conflicts of interest in this work.

\section{References}

1. Bosch FX, Ribes J, Diaz M, Cleries R. Primary liver cancer: worldwide incidence and trends. Gastroenterology. 2004;127:S5-S16.

2. Parikh S, Hyman D. Hepatocellular cancer: a guide for the internist. Am J Med. 2007;120:194-202.

3. El-Serag HB. Hepatocellular carcinoma: an epidemiologic view. J Clin Gastroenterol. 2002;35:S72-S78.

4. Motola-Kuba D, Zamora-Valdes D, Uribe M, Mendez-Sanchez N. Hepatocellular carcinoma. An overview. Ann Hepatol. 2006;5:16-24.

5. Zhang BH, Yang BH, Tang ZY. Randomized controlled trial of screening for hepatocellular carcinoma. J Cancer Res Clin Oncol. 2004;130: $417-422$.

6. Bruix J, Sherman M. Management of hepatocellular carcinoma: an update. Hepatology. 2011;53:1020-1022.

7. European Association For The Study Of The Liver; European Organisation For Research And Treatment Of Cancer. EASL-EORTC clinical practice guidelines: management of hepatocellular carcinoma. J Hepatol. 2012;56:908-943.

8. Benson AB 3rd, Abrams TA, Ben-Josef E, et al. NCCN clinical practice guidelines in oncology: hepatobiliary cancers. J Natl Compr Canc Netw. 2009; 7:350-391.

9. Facciuto ME, Singh MK, Rochon C, et al. Stereotactic body radiation therapy in hepatocellular carcinoma and cirrhosis: evaluation of radiological and pathological response. J Surg Oncol. 2012;105: 692-698.

10. Shrager B, Jibara G, Schwartz M, Roayaie S. Resection of hepatocellular carcinoma without cirrhosis. Ann Surg. 2012;255:1135-1143.

11. Ota K, Teraoka S, Kawai T. Donor difficulties in Japan and Asian countries. Transplant Proc. 1995;27:83-86.

12. Mazziotti A, Grazi GL, Cavallari A. Surgical treatment of hepatocellular carcinoma on cirrhosis: a Western experience. Hepatogastroenterology. 1998;45(suppl 3):1281-1287. 
13. Bruix J, Sherman M. Management of hepatocellular carcinoma. Hepatology. 2005;42:1208-1236.

14. Golfieri R, Cappelli A, Cucchetti A, et al. Efficacy of selective transarterial chemoembolization in inducing tumor necrosis in small $(<5 \mathrm{~cm})$ hepatocellular carcinomas. Hepatology. 2011;53:1580-1589.

15. Bargellini I, Sacco R, Bozzi E, et al. Transarterial chemoembolization in very early and early-stage hepatocellular carcinoma patients excluded from curative treatment: a prospective cohort study. Eur J Radiol. 2012; 81:1173-1178.

16. Hsu KF, Chu CH, Chan DC, et al. Superselective transarterial chemoembolization vs hepatic resection for resectable early-stage hepatocellular carcinoma in patients with Child-Pugh class a liver function. Eur $J$ Radiol. 2012;81:466-471.

17. Moher D, Liberati A, Tetzlaff J, Altman DG. Preferred reporting items for systematic reviews and meta-analyses: the PRISMA statement. Int J Surg. 2010;8:336-341.

18. Wells G, Shea BE, O'Connell D. The Newcastle-Ottawa Scale (NOS) for assessing the quality of nonrandomized studies in meta-analyses. In: 3rd Symposium on Systematic Reviews: Beyond the Basics; 2000:3-5.

19. Mantel N, Haenszel W. Statistical aspects of the analysis of data from retrospective studies of disease. J Natl Cancer Inst. 1959;22: 719-748.

20. DerSimonian R, Laird N. Meta-analysis in clinical trials. Control Clin Trials. 1986;7:177-188.

21. Higgins JP, Thompson SG, Deeks JJ, Altman DG. Measuring inconsistency in meta-analyses. BMJ. 2003;327:557-560.

22. Begg CB, Mazumdar M. Operating characteristics of a rank correlation test for publication bias. Biometrics. 1994;50:1088-1101.

23. Egger M, Davey Smith G, Schneider M, Minder C. Bias in meta-analysis detected by a simple, graphical test. BMJ. 1997;315:629-634.

24. Yang HJ, Lee JH, Lee DH, et al. Small single-nodule hepatocellular carcinoma: comparison of transarterial chemoembolization, radiofrequency ablation, and hepatic resection by using inverse probability weighting. Radiology. 2014;271:909-918.

25. Shi HY, Wang SN, Wang SC, Chuang SC, Chen CM, Lee KT. Preoperative transarterial chemoembolization and resection for hepatocellular carcinoma: a nationwide Taiwan database analysis of long-term outcome predictors. J Surg Oncol. 2014;109:487-493.

26. Lin CT, Hsu KF, Chen TW, et al. Comparing hepatic resection and transarterial chemoembolization for Barcelona Clinic Liver Cancer (BCLC) stage B hepatocellular carcinoma: change for treatment of choice? World J Surg. 2010;34:2155-2161.
27. Sotiropoulos GC, Druhe N, Sgourakis G, et al. Liver transplantation, liver resection, and transarterial chemoembolization for hepatocellular carcinoma in cirrhosis: which is the best oncological approach? Dig Dis Sci. 2009;54:2264-2273.

28. Guo Z, Zhong JH, Jiang JH, Zhang J, Xiang BD, Li LQ. Comparison of survival of patients with BCLC stage A hepatocellular carcinoma after hepatic resection or transarterial chemoembolization: a propensity score-based analysis. Ann Surg Oncol. 2014;21:3069-3076.

29. Yoshimi F, Nagao T, Inoue S, et al. Comparison of hepatectomy and transcatheter arterial chemoembolization for the treatment of hepatocellular carcinoma: necessity for prospective randomized trial. Hepatology. 1992;16:702-706

30. Luo J, Peng ZW, Guo RP, et al. Hepatic resection versus transarterial lipiodol chemoembolization as the initial treatment for large, multiple, and resectable hepatocellular carcinomas: a prospective nonrandomized analysis. Radiology. 2011;259:286-295.

31. Bronowicki JP, Boudjema K, Chone L, et al. Comparison of resection, liver transplantation and transcatheter oily chemoembolization in the treatment of hepatocellular carcinoma. J Hepatol. 1996;24:293-300.

32. Kanematsu T, Matsumata T, Shirabe K, et al. A comparative study of hepatic resection and transcatheter arterial embolization for the treatment of primary hepatocellular carcinoma. Cancer. 1993;71:2181-2186.

33. Liu PH, Lee YH, Hsia CY, et al. Surgical resection versus transarterial chemoembolization for hepatocellular carcinoma with portal vein tumor thrombosis: a propensity score analysis. Ann Surg Oncol. 2014;21: $1825-1833$.

34. Llovet JM, Real MI, Montana X, et al. Arterial embolisation or chemoembolisation versus symptomatic treatment in patients with unresectable hepatocellular carcinoma: a randomised controlled trial. Lancet. 2002;359:1734-1739.

35. O'Suilleabhain CB, Poon RT, Yong JL, Ooi GC, Tso WK, Fan ST. Factors predictive of 5-year survival after transarterial chemoembolization for inoperable hepatocellular carcinoma. Br J Surg. 2003;90: 325-331.

36. Yoshioka H, Sato M, Sonomura T, Terada M, Kishi K, Yoshikawa A. Factors associated with survival exceeding 5 years after transcatheter arterial embolization for hepatocellular carcinoma. Semin Oncol. 1997; 24:S6-S29.

37. Ono Y, Yoshimasu T, Ashikaga R, et al. Long-term results of lipiodoltranscatheter arterial embolization with cisplatin or doxorubicin for unresectable hepatocellular carcinoma. Am J Clin Oncol. 2000;23: 564-568.
Drug Design, Development and Therapy

\section{Publish your work in this journal}

Drug Design, Development and Therapy is an international, peerreviewed open-access journal that spans the spectrum of drug design and development through to clinical applications. Clinical outcomes, patient safety, and programs for the development and effective, safe, and sustained use of medicines are a feature of the journal, which

\section{Dovepress}

has also been accepted for indexing on PubMed Central. The manuscript management system is completely online and includes a very quick and fair peer-review system, which is all easy to use. Visit http://www.dovepress.com/testimonials.php to read real quotes from published authors. 